\title{
SIMPLE AND FAST PROCEDURE TO INCORPORATE DOXORUBICINE IN SMALL UNILAMELLAR LIPOSOMES: EFFECTS ON LIPOSOME SIZE AND ZETA POTENTIAL
}

\author{
MIHAI ADRIAN SOCACIU ${ }^{a}$, ZORITA DIACONEASA ${ }^{\text {b }}$ \\ CARMEN SOCACIU ${ }^{\mathrm{b}, \mathrm{c}, *}$
}

\begin{abstract}
This study aimed to find alternative, cheap and fast procedures to incorporate Doxorubicin in small unilamellar nanoliposomes made of Dipalmitoyl Phosphatidyl Choline and Cholesterol. It was compared the entrapment efficiency of doxorubicin in "home-made" liposomes (1) and in ImmunoSOME liposomes (2), a commercial, expensive formulation. It was obtained an entrapment efficiency of $27.6 \%$ vs $12.1 \%$ for formulatios (1) vs (2). The liposomes' size and zeta-potential of formulation (1) ranged from 116 and $120 \mathrm{~nm}$, being smaller than in formulation (2). The zeta potential was negative for both formulations and showed a better stability and monodispersibility also for formulation (1). This fast procedure is convenient and assure efficient doxorubicin entrapment in the monodisperse nanoliposomes. Such simple formulations, easier to be obtained, can offer also good quality/ price ratios and can be produced at larger scale, with higher incorporation efficiency for experimental in vivo studies which aims the targeted delivery of Doxorubicin to cancer tumors in animals.
\end{abstract}

Keywords: Liposomes, Doxorubicin encapsulation, size and zeta potential

a University of Medicine and Pharmacy "Iuliu Haţieganu" 12, Victor Babeş str., RO-400028, Cluj-Napoca, Romania

b University of Agricultural Sciences and Veterinary Medicine, 3-5 Mănăştur str., RO-400372 Cluj-Napoca, Romania

${ }^{c}$ Research Center for Applied Biotechnolgy in Diagnosis and Molecular Therapy, BIODIATECH, str. Trifoiului 12G, 400478 Cluj-Napoca, Romania

*Corresponding author: carmen.socaciu@usamvcluj.ro 


\section{INTRODUCTION}

Anthracyclines such as doxorubicin (DOXO), epirubicin and daunorubicin are among the most active cytotoxic agents, a highly potent chemotherapeutic agent for the treatment of a wide variety of solid tumors and hematological malignancies. Since the secondary effects associated with their chronic administration induce cardiomyopathy and congestive heart failure, new, less toxic formulations (pegylated or nonpegylated liposomal formulations) demonstrated the superiority of liposomal doxorubicin in comparison to conventional non-liposomal doxorubicin. Therefore, liposomal formulations with entrapped DOXO are currently the best-known alternatives to improve the index and spectrum of anticancer activity and decrease cardiotoxicity [1].

Liposomes are prepared, characterized and used since decades as potent drug delivery carriers, being largely characterized for their physicochemical properties, highly dependent on their qualitative and quantitative phospholipid composition, pH, size [2-5]. Mostly used is Dipalmitoyl Phosphatidyl Choline (DPPC) in combination with other types of phospholipids and in different ratios with cholesterol, which confers stability and modulate membrane fluidity $[6,7]$

It is well documented, that drug delivery using carriers like liposomes provides several advantages, e.g. increased solubility, improved tumor targeting, enhanced accumulation in tumor tissues and cells, decreased systemic toxicity and increased maximum tolerated dosage [8, 9].

Nowadays, new formulations containing doxorubicin encapsulated in liposomes are available and produced, at high prices, by some specialized companies. For example, Doxosome is a DOXO-entraped liposomal suspension and Doxil, commercially known as Caelyx, is a Polyethyleneglycolcoated liposomal formulation including DOXO, used for the treatment of ovarian cancer, sarcoma and multiple myeloma. In spite of showing reduced DOXO-related toxicity, Doxil showed a lower clinical efficiency, since the PEGylated liposomal doxorubicin was sequestered in the cellular lysosomes, with a lower bioavailability of DOXO [10]. Another comparative study of the antitumor activity of free DOXO and Doxil in mouse lymphoma models concluded contrasting effects, e.g. free DOXO was more efficient by i.p administration while Doxil was superior by i.v. administration [11]. Another commercial formulation is ImmunoSOME, a surface reactive product without DOXO, which include PEG-conjugated liposomes linked to biotin. This product offers possibilities to link avidine-conjugated microbubble and target tumors by ultrasounds (US), recommending DOXO-liposomes as good nanotheranostic agents [12]. 
In this context, our experimental study aimed to find a fast and cheap procedure to incorporate DOXO in small unilamellar liposomes (SUV) as simple formulations made of Dipalmitoyl Phosphatidyl Choline (DPPC) and Cholesterol (CHOL) (1). Comparatively, ImmunoSOME, an expensive commercial PEGylated liposomal suspension was used to incorporate similar quantities of DOXO (2). The results were monitored by measuring the DOXO entrapment efficiency (EE), the liposomes' size and zeta-potentials, after DOXO incorporation in both formulations (1) and (2).

\section{RESULTS AND DISCUSSION}

\section{Evaluation of DOXO entrapment efficiency}

The influence of liposomal lipid composition on liposome size, zeta potential and liposome-induced dendritic cell maturation using a design of experiments approach was recently reported [13]. Another novel approach on drug delivery refers to the key-role of the lipid composition and nanoformulation of liposomal DOXO in relation to the biological evaluation of entrapped DOXO [14]. The antiproliferative long-circulating liposomes coencapsulating DOXO and curcumin, through the use of a quality-by-design approach was also reported, recently. Six parameters, namely the phospholipid, curcumin and DOXO concentrations, the phospholipid: cholesterol molar ratios, the temperature during evaporation, buffer's $\mathrm{pH}$ and hydration steps were identified as potential risk factors for the quality of the final product. The influence of these variables was monitored by the particle size, zeta potential, drug loading and entrapment efficiency [15].

To intensify the accumulation of DOXO inside SUV vs ImmunoSOME liposomes, we tried either the classical procedure using only Hepes buffer at $\mathrm{pH} 7.4$ (a) or a combination of Hepes buffer and gradients of ammonium sulfate to obtain a more 'active' loading of amphipathic weak bases into the aqueous compartment of liposomes (b), approach being applied previously to encapsulate anthracyclines inside the liposomes at very high efficiency [16]. The results were superior in our case using procedure (b) and are presented in Table 1 which includes the comparative values (mg of DOXO added to liposomal suspension (DOXOadded), DOXO found in the supernatant (DOXOout), found in the pellet (DOXOin, calculated as the difference DOXO added - DOXOout (column 4) or by direct determination after the dissolution of the pellet (DOXOin* - column 5). The encapsulation efficiency (EE) was calculated as a ratio DOXOin*/DOXOadded. 
Table 1. The encapsulation efficiency (EE) of DOXO inside liposomes, taking into account the DOXO before and after encapsulation.

For sample abbreviations see the Experimental Section.

\begin{tabular}{|l|c|c|c|c|c|}
\hline \multicolumn{1}{|c|}{ Sample } & $\begin{array}{c}\text { DOXO } \\
\text { added } \\
\text { (mg) }\end{array}$ & $\begin{array}{c}\text { DOXOout } \\
\text { (mg) }\end{array}$ & $\begin{array}{c}\text { DOXOin } \\
\text { (calculated) } \\
\text { (mg) }\end{array}$ & $\begin{array}{c}\text { DOXOin* } \\
\text { (determined) } \\
\text { (mg) }\end{array}$ & EE (\%) \\
\hline L1 (SUV) & 1 & 0.73 & 0.27 & 0.276 & 27.60 \\
\hline L2 (SUV) & 1.4 & 1.31 & 0.09 & 0.068 & 4.85 \\
\hline L3(ImmunoSOME) & 1.4 & 1.28 & 0.12 & 0.110 & 7.85 \\
\hline L4(ImmunoSOME) & 1 & 0.86 & 0.14 & 0.121 & 12.1 \\
\hline L5(ImmunoSOME) & 1.4 & 1.24 & 0.16 & 0.125 & 8.92 \\
\hline L6 (SUV) & 1.4 & 0.89 & 0.51 & 0.375 & 26.78 \\
\hline
\end{tabular}

Significant differences were observed between the six samples: The SUV liposomes (L1 and L6) showed higher incorporation yields than ImmunoSOME. When sucrose was added, the incorporation decreased significantly $(\mathrm{L} 2<\mathrm{L} 1$ and $\mathrm{L} 3<\mathrm{L} 5)$. No significant differences between $\mathrm{EE}$ values were observed when DOXO was added as 1 or $1.4 \mathrm{mg}$ (see L1 vs L6), suggesting that the liposomal membrane structure is mainly responsible for the limitations of DOXO incorporation.

The more complex composition of ImmunoSOME membrane determined a limited incorporation of DOXO, at around $50 \%$ comparing to SUV liposomes (L4 vs L1).

\section{Liposomes Size and Polydispersibility index}

Fig. 1 shows the values of liposome sizes for samples L1 (SUV-DOXO liposomes), comparative to L3, L4, L5 (ImmunoSOME-DOXO liposomes).

The DOXO-SUV liposomes from samples L1 and L6 had similar sizes, of 116 and $120 \mathrm{~nm}$, respectively. Also, their polydispersity index (PDI) is good (0.162-0.170), with values less than 0.2 , suggesting a good monodispersion of liposomes. For samples containing DOXO- ImmunoSOME liposomes (L3, L4 and L5) the sizes ranged from 148 to $163 \mathrm{~nm}$ (larger size), and PDI values from 0.119 to 0.214 (still monodisperse). 


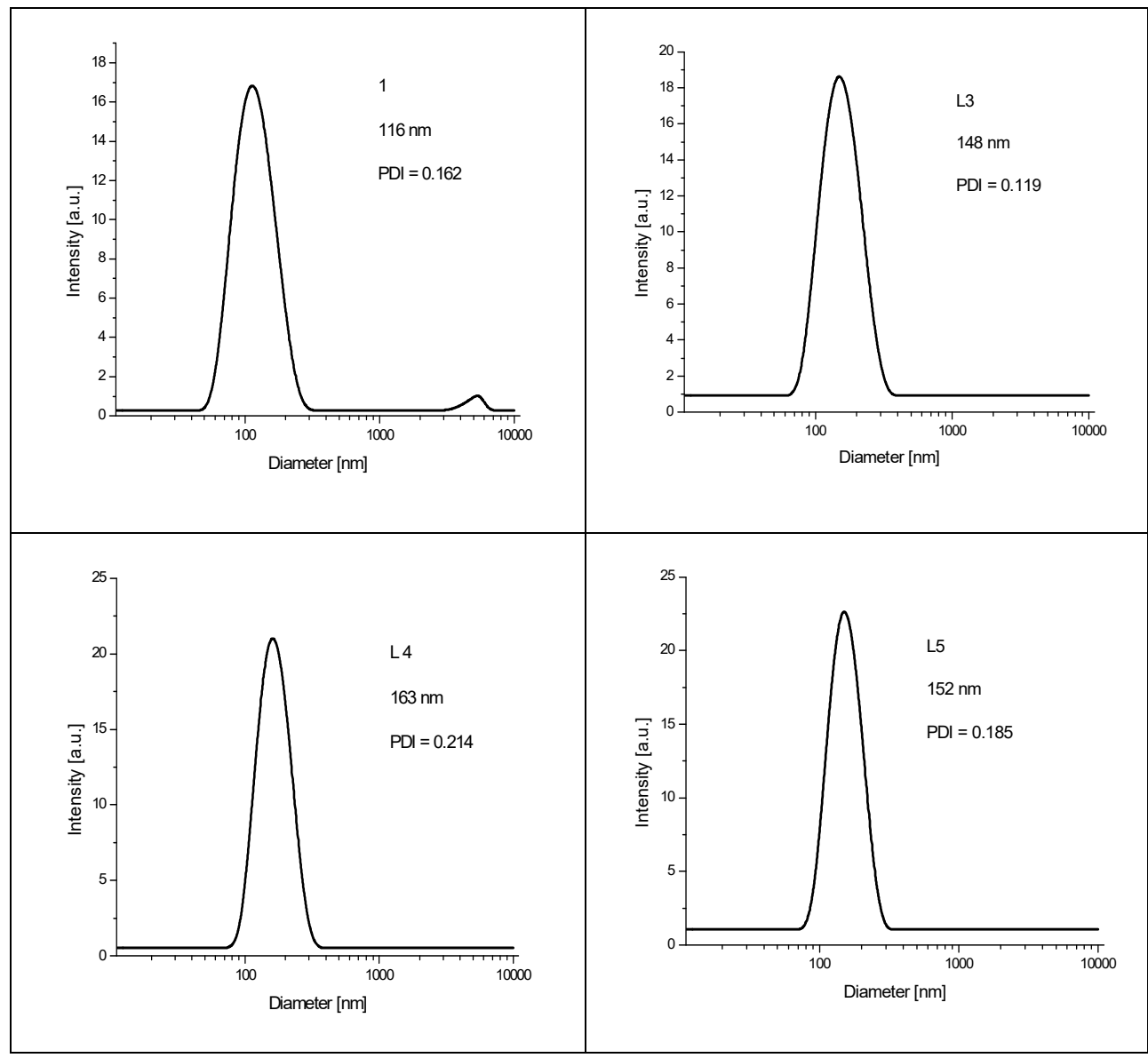

Figure 1. The liposome size for samples L1 (SUV-DOXO liposomes), comparative to L3,L4, L5 (ImmunoSOME-DOXO liposomes)

\section{Zeta-potential of DOXO liposomes}

The measurements of zeta potential of the six samples ( $\mathrm{L} 1$ and L6 containing DOXO-SUV liposomes and L3-L5 containing DOXO-ImmunoSOME liposomes) showed negative values in all cases, as shown in Fig.2.

The zeta potential of the SUV-DOXO and ImmunoSOME-DOXO liposomes was negative (ranging from -26 to $-48 \mathrm{mV}$ ), due to their charge and $\mathrm{pH}$ of the buffer, The higher values were noticed for $\mathrm{L} 1(-48 \mathrm{mV})$ suggesting the best stability, followed by L2 and L6 ( -30 and $-26 \mathrm{mV}$, respectively). The samples L3-L5 corresponding to DOXO-ImmunoSOME liposomes had closer values, from -27 to $-29 \mathrm{mV}$. 


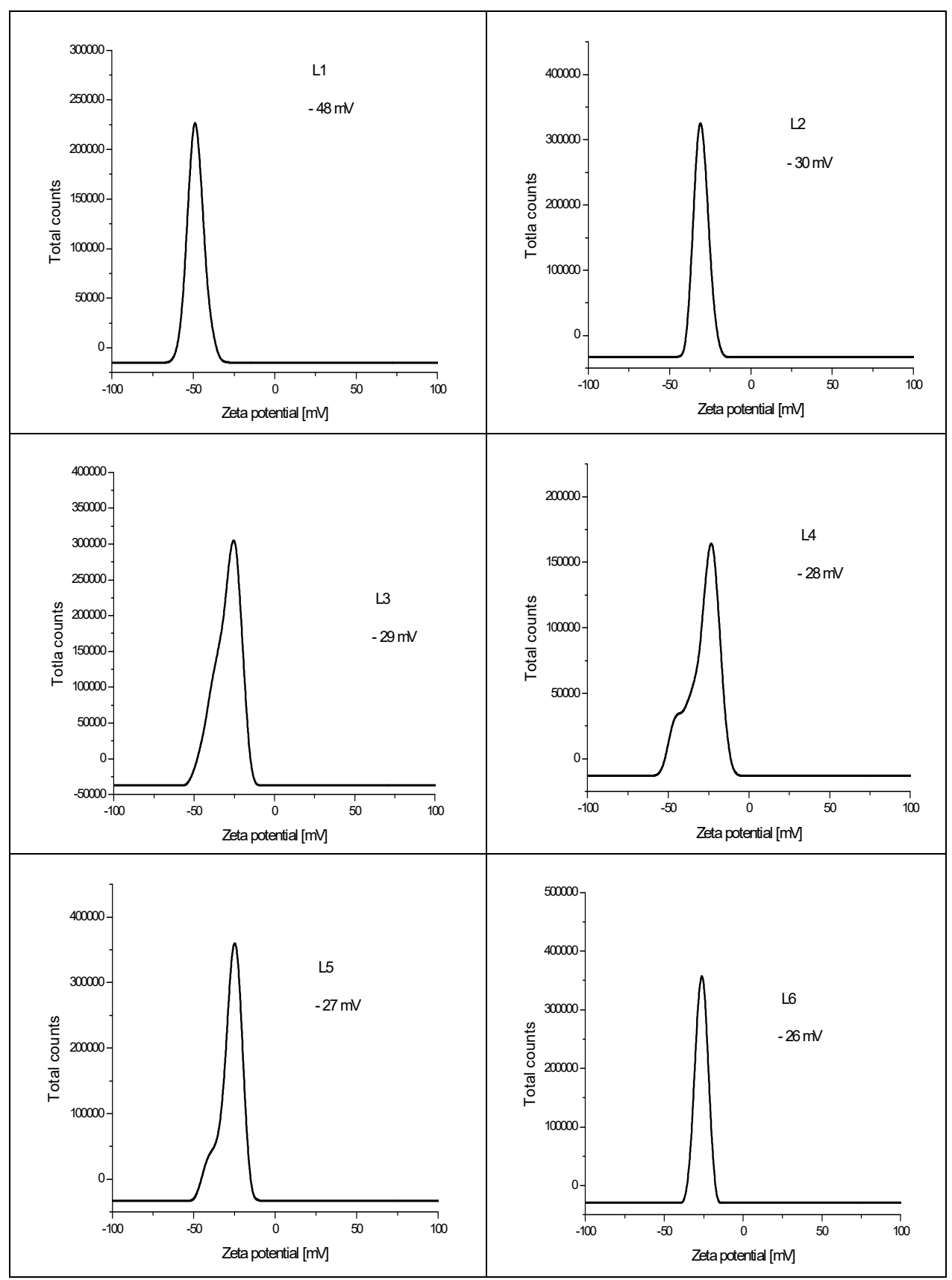

Figure 2. The values of zeta potential for the six samples (L1 to L6). For abbreviations see the Experimental Section Table 2. 
The significance of the zeta potential is related to the short- and longterm stability of emulsions and liposomal suspensions. Zeta potential becomes more negative when $\mathrm{pH}$ increases and is a measure of magnitude of charges on liposomal vesicles. Recently such assumptions were demonstrated for DPPC and 1,2-dioleoyl-sn-glycero-3-phosphatidylcholine (DOPC) liposomes investigated in $1 \mathrm{mM} \mathrm{NaCl}(\mathrm{pH} 6.2)$ and phosphate buffer $(\mathrm{pH} 8.1)$ in relation to the liposome size, polydispersity index, and zeta potentials [17]. The higher the value of the zeta potential is (positive or negative), the more stable the colloid dispersion is. Normally, a value of $>30 \mathrm{mV}$ indicates good stability [17]. Our data are in agreement with recent reports which demonstrated the superiority of liposomal doxorubicin in comparison to conventional non-liposomal doxorubicin [18], especially in SUV formulations with high zeta potentials.

Considering these data, sample L1 (SUV-DOXO liposomes) showed best stability and dispersibility. The ImmunoSOME-DOXO samples had inferior properties comparing to SUV-DOXO.

\section{CONCLUSION}

The results of this study show that simple and fast procedures can be applied to incorporate DOXO in "home-made" SUV-unilamellar nanoliposomes containing cheap and simple lipid composition. We have shown that entrapment efficiency of DOXO into SUV-liposomes (L1) was higher than into ImmunoSOME liposomes (L4), having values of $27.6 \%$ vs $12.1 \%$ respectively, when the same initial DOXO quantity was added.

The addition of sucrose decreased significantly the entrapment efficiency, but no significant differences.

Meanwhile, no significant differences between the entrapment efficiency were observed when DOXO was added as 1 or $1.4 \mathrm{mg}$, suggesting that the liposomal membrane structure is mainly responsible for the limitations of DOXO incorporation.

The more complex composition of ImmunoSOME membrane may explain the lower incorporation rate of DOXO, at around $50 \%$ comparing to SUV liposomes. This is an expensive commercial liposomal formulation, using the transmembrane ammonium ion gradient procedure and its complex structure seems to hinder the DOXO incorporation.

Considering the liposomes' size and the zeta potential, SUV-DOXO liposomes showed best stability and dispersibility and is recommended to be obtained at larger scale by this simple, cheap and fast procedure. 
Finally, we consider this simple and fast procedure to prepare SUVDOXO liposomes can be upgraded, in order to obtain higher quantities of this liposomal suspension. Such simple formulations with high entrapment efficiency of DOXO may offer good quality/price ratios and can be produced at larger scale for experimental studies in vivo, for a targeted delivery to cancer tumors in small animals.

\section{EXPERIMENTAL SECTION}

\section{Chemicals, reagents and materials}

1,2-dipalmitoyl sn-glycero-3-phosphatidylcholine (DPPC, MW=734), Cholesterol (Roth puritate 99\%, (CHO L, MW=386), Doxorubicin clorhydrate (DOXO) $2 \mathrm{mg} / \mathrm{ml}$ saline solution, ImmunoSOME (5 ml, Encapsula Nanoscienes) a mixture of HSPC:CHOL:DSPE-PEG: DSPE-PEG-Biotin (55:40:4:1) representing $5.96 \mathrm{mg}$ lipids/ml in $100 \mathrm{~nm}$ liposomes. Buffer HEPES $10 \mathrm{mM}$ (pH 7.4), buffer Ammonium Sulphate pH 5.5 (Sigma-Aldrich).

\section{Instrumentation}

BENACOR rotavapor with automatic regulation of vacuum, temperature and rotation, ultrasonic generator UP200St-G (Hielscher Ultrasonics $\mathrm{GmbH}$ ) $200 \mathrm{~W}, 26 \mathrm{kHz}$ with an S26d2 sonotrode of $2 \mathrm{~mm}$ and ultrasonic bath, thermostate with recirculated water, extrusion system of LiposoFast-50, criocentrifuge Hettich 460R, Eppendorf centrifuge (max. 15000 rpm), Cell Media mixer. The spectrophotometric measurements were made on a Perkin Elmer UV WinLab Spectrophotometer (200-700 $\mathrm{nm}$ at $1 \mathrm{~nm} / \mathrm{min})$.

\section{Preparation of Unilamellar liposomes}

To a mixture of $375 \mathrm{mg}$ lecithin and $125 \mathrm{mg}$ cholesterol, in a molar ratio 61.5:38.5 it was added a solvent mixture of chloroform:methanol 2:1 (v:v), in a glass round bottom flask of $50 \mathrm{ml}$. The solvent was evaporated under vacuum in the Rotavapor at $43^{\circ} \mathrm{C}$, for $30 \mathrm{~min}$. The lipid layer was then dehydrated in an exicator containing anhydrous Calcium chloride, during 48 hrs. The lipid layer was suspended in $20 \mathrm{ml}\left(\mathrm{NH}_{4}\right)_{2} \mathrm{SO}_{4} 300 \mathrm{mM}, \mathrm{pH} 5.5$, and strongly vortexed. The liposome suspension was taken from the flask and centrifuged at $4^{\circ} \mathrm{C}, 4600 \mathrm{rpm}, 20 \mathrm{~min}$. The upper, opalescent phase containing multilamellar liposomes was retained and extruded successively through 
polycarbonate filters of 400 and $100 \mathrm{~nm}$, using the Lipofast system. The extruded suspension contained small unilamellar liposomes (SUV), whose size was determined, as mentioned below.

\section{Incorporation of DOXO in SUV and ImmunoSOME liposomes}

The incorporation of DOXO in unilamellar liposomes was realized by the transmembrane ammonium ion gradient procedure, as follows: under mix in the ultrasonic bath at $65^{\circ} \mathrm{C}, 30 \mathrm{~min}$, to a volume of $0.3 \mathrm{ml}$ liposome suspensions (SUV or ImmunoSOME) there were added 0.5 or $0.7 \mathrm{ml}$ DOXO and $0.5 \mathrm{ml} \mathrm{Hepes} 10 \mathrm{mM} \mathrm{pH} \mathrm{7.4.} \mathrm{as} \mathrm{mentioned} \mathrm{in} \mathrm{Table} \mathrm{2.} \mathrm{The} \mathrm{mixture} \mathrm{was}$ maintained $10 \mathrm{~min}$ at $4^{\circ} \mathrm{C}$ in the refrigerator, then a volume of $0.8 \mathrm{Ammonium}$ sulphate $300 \mathrm{mM} \mathrm{pH} 5.5$ was added to the suspension, kept $10 \mathrm{~min}$ at room temperature and centrifuged at $15000 \mathrm{rpm}, 10 \mathrm{~min}$. The supernatant was separated and the DOXOout (the non-incorporated DOXO) was determined by UV-Vis spectrometry. The pellet was washed with Hepes buffer two times and then re-suspended in $0.7 \mathrm{ml}$ Hepes $\mathrm{pH} 7.4$, mixed for $15 \mathrm{~min}$ at $45^{\circ} \mathrm{C}$.

Fig. 3 reflect the differences between the two types of liposomes used for DOXO entrapment, as well the mechanisms behind the DOXO loading using a transmembrane ammonium ion gradient in the unilamellar liposomes.

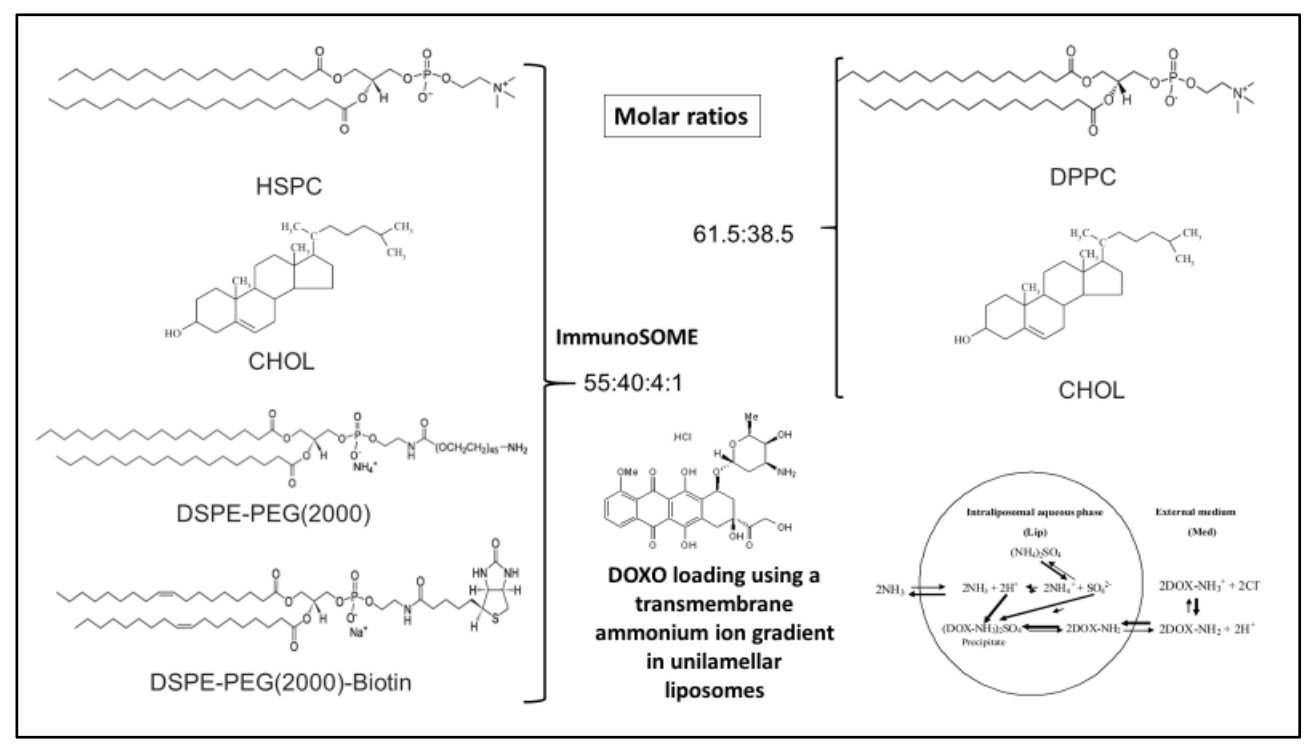

Figure 3. The composition of the SUV and ImmunoSOME liposomes used for DOXO entrapment and the mechanisms behind the DOXO loading using a transmembrane ammonium ion gradient. 
Table 2 represents the volumes of liposome suspensions (SUV and ImmunoSOME) and Hepes buffer, with or without addition of sucrose, used to incorporate DOXO (volumes of 0.5 or $0.7 \mathrm{ml}$ representing 1 and $1.4 \mathrm{mg}$, respectively).

Table 2. Incorporation of DOXO (using 0.5 or $0.7 \mathrm{ml}$ from a solution of $2 \mathrm{mg} / \mathrm{ml}$ ) into SUV or ImmunoSOME liposomes, in $0.5 \mathrm{ml} \mathrm{Hepes} \mathrm{buffer} 10 \mathrm{mM}(\mathrm{pH} 7.4)$, with or without sucrose $10 \%$.

\begin{tabular}{|l|l|l|l|}
\hline Abbreviation & Liposome type and volume & DOXO (ml) & $\begin{array}{c}+/- \text { sucrose } \mathbf{1 0 \%} \\
(\mathbf{m l})\end{array}$ \\
\hline L1 & $0.3 \mathrm{ml} \mathrm{SUV}$ & 0.5 & - \\
\hline L2 & $0.3 \mathrm{ml} \mathrm{SUV}$ & 0.7 & $2 \mathrm{ml}$ sucrose \\
\hline L3 & $0.3 \mathrm{ml} \mathrm{ImmunoSOME}$ & 0.7 & $2 \mathrm{ml}$ sucrose \\
\hline L4 & $0.3 \mathrm{ml}$ ImmunoSOME & 0.5 & - \\
\hline L5 & $0.3 \mathrm{ml}$ ImmunoSOME & 0.7 & - \\
\hline L6 & $0.3 \mathrm{ml} \mathrm{SUV}$ & 0.7 & - \\
\hline
\end{tabular}

After the incorporation procedure, a volume of $0.5 \mathrm{ml}$ from each final suspension was taken to determine the liposomes size and zeta potential > concomitantly, to $0.2 \mathrm{ml}$ suspension, a volume of $0.1 \mathrm{ml}$ methanol and $0.8 \mathrm{ml}$ Hepes buffer was added and the absorption intensity was recorded at 480 $\mathrm{nm}$, in order to evaluate the DOXO incorporated in liposomes (DOXOin). In parallel, the empty liposomes were dissolved, and considered as blank.

\section{Evaluation of DOXO entraped in SUV or ImmunoSOME liposomes}

First, a calibration curve was built, using different dilutions of the initial DOXO solution $(2 \mathrm{mg} / \mathrm{ml})$, the curve factor $\left(f=c / A_{480 \mathrm{~nm}}\right)$ having the value $\mathrm{f}=0.133$.

The formula used to calculate the quantity of DOXOin and DOXOout was the following:

DOXO $(\mathrm{mg})=\mathrm{f} x$ dilution factor $x$ total volume of the solution collected (from supernatant or dissolved liposomes containing DOXO).

\section{Evaluation of liposomes' size and zeta potential}

The liposome hydrodynamic diameter and polydispersity index (PDI) were determined at $25^{\circ} \mathrm{C}$ by dynamic light scattering (DLS) using a Malvern Zetasizer Nano ZS-90 (Malvern Instruments) with a He-Ne laser operating at a wavelength of $633 \mathrm{~nm}$ and an avalanche photodiode detector. The zeta 
SIMPLE AND FAST PROCEDURE TO INCORPORATE DOXORUBICINE IN SMALL UNILAMELLAR LIPOSOMES: EFFECTS ON LIPOSOME SIZE AND ZETA POTENTIAL

potential of the liposomes was determined also at $25^{\circ} \mathrm{C}$ by the laser Doppler microelectrophoresis technique using the same Malvern equipment. Before measurements, the samples were diluted 5 fold with ultrapure water.

\section{ACKNOWLEDGMENTS}

This study was supported by the Grant nr. 4945/12/2016 funded by the University of Medicine and Pharmacy "Iuliu Haţieganu" Cluj-Napoca. The authors would like to thank Prof. Dr. Simion Aştilean and Dr. Monica Potara (Interdisciplinary Research Institute in Bio-Nano-Sciences, University "Babeş-Bolyai") for their kind support and help related to the measurements of liposomes'size and zeta potential.

\section{REFERENCES}

1. H. Cortes-Funes; C. Coronado; Cardiovasc. Toxicol., 2007, 7, 56-60.

2. A. Akbarzadeh; R. Rezaei-Sadabady; S. Davaran; S.W. Joo; N. Zarghami; Y. Hanifehpour; M. Samei; M. Kouhi; K. NejatiKoshki; Nanoscale Res. Lett., 2013, 8, 1-9.

3. N. Bergstrand; PhD diss. Acta Univ. Upsaliensis, Uppsala, 2003.

4. J.S. Dual; A.C. Rana; A.K. Bhandari; Int. J. Pharm. Sci. Res., 2012, 3, 14-20.

5. Y. P. Patil; S.Jadhav; Chem. Phys. Lipids, 2014,177, 8-18.

6. H. Ohvo-Rekila; B. Ramstedt; P. Leppimaki; J.P. Slotte; Prog. Lipid Res., 2002, 41, 66-97.

7. C. Socaciu; R. Jessel; H. Diehl; Chem. Phys. Lipids, 2000, 106, 79-88.

8. A. Gabizon; R. Shiota; D. Papahadjopuulos; J. Natl. Cancer I., 1989, 81,14841488.

9. A. Gabizon; R. Catane; B. Uziely; B. Kaufman; T. Safra; R. Cohen; Cancer Res., 1994, 54, 987-992.

10. A.L. Seynhaeve; B.M. Dicheva; S. Hoving; G.A. Koning; T.L. Ten Hagen; J. Control. Release, 2013, 172, 330-340.

11. Cabanes A; Tzemach D; Goren D; Horowitz AT; Gabizon A. Clin. Cancer Res., 1998, 4, 499-505.

12. P. Mohan; N.Rapoport; Mol. Pharm. 2010, 7, 1959-1973.

13. P. C. Soema; G-J. Willems; W. Jiskoot; J-P. Amorij; G.F. Kersten; Eur. J. Pharm. Biopharm., 2015, 94, 427-435.

14. F. Haghiralsadat; G. Amoabediny; M.H. Sheikhha; T. Forouzanfar; J.M. Arough; M.N. Helder; B. Zandieh-doulabi; Cell Journal (Yakhteh), 2017, 19, 55-65. 
15. L.R. Tefas; B. Sylvester; I. Tomuta; A. Sesarman; E. Licarete; M. Banciu; Drug Des. Dev. Ther., 2017, 11, 1605-1621.

16. G. Haran; R. Cohen; L.K. Bar; Y. Barenholz; Biochim. Biophys. Acta, 1993, 1151, 201-215.

17. E. Chibowski; A. Szczes; Adsorption, 2016, 22, 755-765.

18. Y.H. Ngan; M. Gupta; Arch. Pharm. Pract., 2016, 7, 1-13. 